\title{
MOVING TARGETS IN SYNTHETIC APERTURE IMAGES: A BAYESIAN APPROACH*
}

\author{
Paulo A. C. Marques \\ ISEL-DEEC \\ R. Conselheiro Emídio Navarro 1, \\ 1949-014 Lisboa Portugal \\ E-mail: pmarques@isel.pt
}

\begin{abstract}
This paper presents a novel method to determine the complete velocity vector of a moving target using a single Synthetic Aperture Radar (SAR) sensor. The method exploits the structure of the returned echo from a moving target: in the slow-time frequency domain, it is a scaled and shifted replica of the antenna radiation pattern immersed in Gaussian noise; the scale and the shift are related with the slant-range and the cross-range velocities, respectively. A Bayesian approach is then adopted to derive an estimator for the velocity vector. Simulation results illustrating the estimator effectiveness are presented.
\end{abstract}

\section{INTRODUCTION}

Figure 1 shows a typical Synthetic Aperture Radar (SAR) geometry. As the radar travels at constant velocity in azimuth direction (navigation direction), short microwave pulses are transmitted at regular intervals, and the corresponding echoes are recorded. High resolution in azimuth is achieved by synthesizing a large aperture, exploiting the relative motion between the platform and the ground. If targets are moving, the SAR image will show them defocused and/or at wrong positions, depending on their motion direction [1], [2]. A moving target in the azimuth direction appear blurred whereas one that moves on the range direction appears misplaced. Several methods have recently been proposed to detect, image and estimate moving targets trajectory parameters (see for instance [3], [4]). These works have not taken into account the antenna radiation pattern, which is equivalent to assuming an omnidirectional antenna radiation pattern. In this case, the so-called blind angle ambiguity limitation occurs: unless stereo measures are available, it is not possible to determine the complete velocity vector, but only its magnitude [3].

In this work it is shown that it is possible to infer the complete velocity vector of a moving target using data from

*THIS WORK WAS SUPPORTED BY PORTUGUESE PRAXIS XXI PROGRAM, UNDER PROJECT 2/2.1.TIT/1580/95.
José M. B. Dias

\author{
IST-IT \\ Torre Norte, Piso 10, Av. Rovisco Pais, \\ 1049-001 Lisboa Portugal \\ E-mail: bioucas@1x.it.pt
}

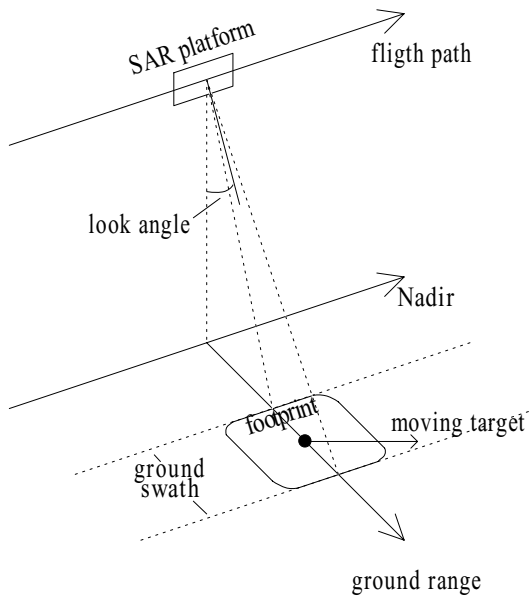

Fig. 1. Typical SAR geometry.

a single sensor if the antenna radiation pattern is known. We show that the returned signal from a moving target, in the slow-time frequency domain, is a scaled and shifted replica of the antenna radiation pattern immersed in Gaussian noise. A Bayesian approach is taken to derive an optimal estimator of the velocity parameters. By adopting suitable noninformative prior, the target reflectivity, which is a nuisance parameter, is integrated out.

The paper is organized as follows: Section 2 considers the effects of moving targets on the received data. In Section 3 a Bayesian approach is employed and the maximum a posteriori probability (MAP) is adopted as estimation criterion. Section 4 presents simulation results illustrating the estimator performance.

\section{MOVING TARGETS IN SAR IMAGERY}

Consider the SAR geometry sketched in Fig. 2, where the radar platform travels at speed $v_{r}$ in the navigation direc- 


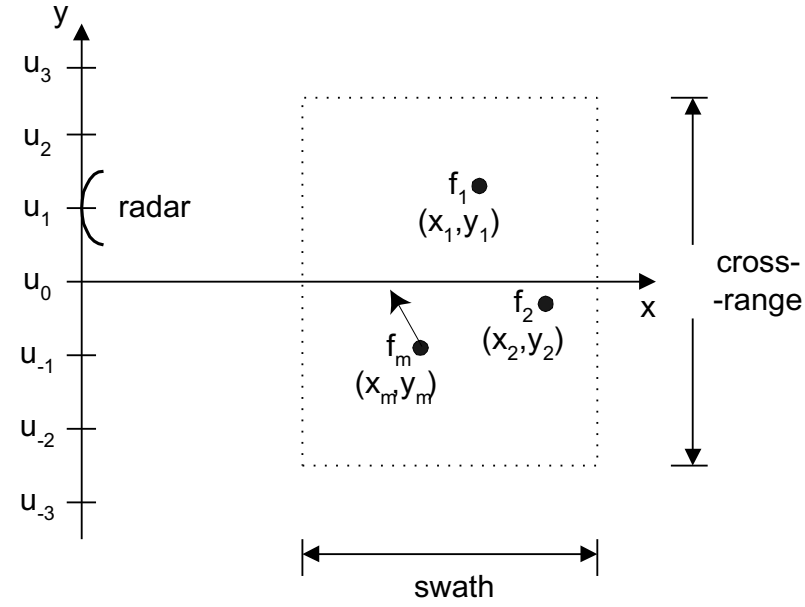

Fig. 2. SAR geometry in the slant-range plane.

tion and has an antenna radiation pattern $a$, which is function of the observation angle. Consider a single moving target with constant complex reflectivity $f_{m}$, coordinates $\left(x_{m}, y_{m}\right)$ when the platform is at position $y=0$, and speed vector $\left(v_{x}, v_{y}\right) \equiv\left(\mu_{m} v_{r}, b_{m} v_{r}\right)$ defined in the slant-plane $(x, y)$. Vector $\left(\mu_{m}, b_{m}\right)$ denotes the target relative velocity with respect to the radar. When the radar is positioned at the coordinate $y=u$, the corresponding received signal after quadrature demodulation and in the fast-time frequency domain is (see, [4] for details)

$$
\begin{array}{r}
s_{m}(u, \omega)=a\left(x_{m}-\mu_{m} u, y_{m}-\nu_{m} u\right) \times \\
P(\omega) f_{m} e^{-j 2 k \sqrt{\left.X_{m}^{2}+\left(Y_{m}-\alpha_{m} u\right)^{2}\right)}},
\end{array}
$$

where $P(\omega)$ is the Fourier transform of the transmitted signal, $k \equiv 2 \pi / \lambda$ is the wavenumber, $\nu_{m} \equiv 1+b_{m}, \alpha_{m} \equiv$ $\sqrt{\mu_{m}^{2}+\nu_{m}^{2}}$, and $X_{m}$ and $Y_{m}$ are the so-called generalized coordinates for the target motion parameters [5], satisfying

$$
\begin{aligned}
\alpha_{m} Y_{m} & \equiv \mu_{m} x_{n}+\nu_{m} y_{m} \\
r_{m} & \equiv \sqrt{x_{m}^{2}+y_{m}^{2}} \equiv \sqrt{X_{m}^{2}+Y_{m}^{2}}
\end{aligned}
$$

By applying the stationary phase method [3] to $s_{m}(u, \omega)$ with respect to $u$, we obtain

$$
\begin{aligned}
S_{m}\left(k_{u}, \omega\right) & =P(\omega) A\left(k_{u}, \boldsymbol{\theta}\right) \\
& \times f_{m} e^{-j \sqrt{4 k^{2}-\left(\frac{k_{u}}{\alpha_{m}}\right)^{2}} X_{m}} e^{-j \frac{k_{u}}{\alpha_{m}} Y_{m}},
\end{aligned}
$$

where $\boldsymbol{\theta} \equiv\left(\mu_{m}, \nu_{m}\right)$ denotes the velocity vector parameter, $A\left(k_{u}, \boldsymbol{\theta}\right)=a\left(x_{m}-\mu_{m} u\left(k_{u}\right), y_{m}-\nu_{m} u\left(k_{u}\right)\right)$, and (see [?] for details)

$$
k_{u}=2 k \frac{\alpha_{m}\left(Y_{m}-\alpha_{m} u\right)}{\sqrt{X_{m}^{2}+\left(Y_{m}-\alpha_{m} u\right)^{2}}} .
$$

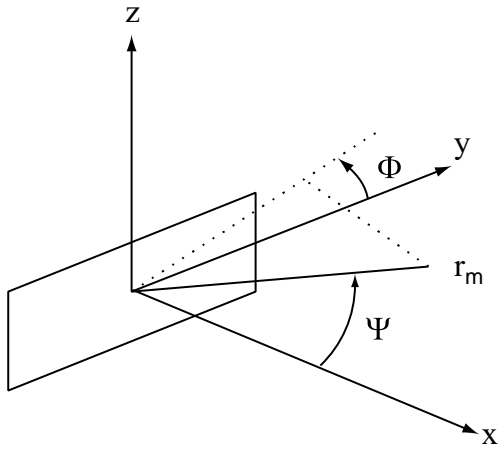

Fig. 3. Antena aperture in the $x=0$ plane.

Given that the irradiated field from a planar aperture is [6]

$$
A\left(k_{u}, \boldsymbol{\theta}\right) \propto f\left(k_{0} \sin \psi \cos \phi, k_{0} \sin \psi \sin \phi\right),
$$

where $\psi$ and $\phi$ are the angles shown in Fig. 3 and $f\left(k_{u}, k_{x}\right)$ is the Fourier transform of the aperture field computed at $k_{u}=k_{0} \sin \psi \cos \phi$, and $k_{x}=k_{0} \sin \psi \sin \phi$, the following relation holds, after some algebraic manipulation of equations (2), (3), and (5):

$$
k_{0} \sin \psi \cos \phi \approx \frac{1}{2 \nu_{m}}\left(k_{u}-2 k \mu_{m}\right) .
$$

Assuming that the illumination does not exhibit relevant fluctuations in range direction, one is lead to

$$
A\left(k_{u}, \boldsymbol{\theta}\right) \propto f\left[\frac{1}{2 \nu_{m}}\left(k_{u}-2 k \mu_{m}\right), 0\right] .
$$

Hence, the illumination function in the slow-time frequency domain takes, approximately, the shape of the antenna radiation pattern in the cross-range direction. The shape becomes shifted by $2 k \mu_{m}$ and is compressed by $2 \nu_{m}$. The shift depends on the target slant-range velocity and the expansion depends on the target cross-range velocity. This result suggests that it is possible to solve the blind angle ambiguity using a single radar, if the antenna radiation pattern is known.

\section{MOVING TARGETS DETECTION ESTIMATION AND IMAGING}

Assume that there is only a single moving target. The returned echo is therefore given by

$$
\begin{aligned}
S\left(k_{u}, \omega\right)= & P(\omega) A\left(k_{u}, \boldsymbol{\theta}\right) f_{m} e^{-j \sqrt{4 k^{2}-\left(\frac{k_{u}}{\alpha_{m}}\right)^{2}} X_{m}} e^{-j \frac{k_{u}}{\alpha_{m}} Y_{m}} \\
& +P(\omega) A\left(k_{u}, \boldsymbol{\theta}_{0}\right) \sum_{n} f_{n} e^{-j \sqrt{4 k^{2}-k_{u}^{2}} X_{n}} e^{-j k_{u} Y_{n}},
\end{aligned}
$$

where the first term on the right hand side is due to the moving target and the remaining terms are due to the static 
ground. The reflectivity of all targets is assumed to be independent of the aspect angle. Vector $\boldsymbol{\theta}_{0} \equiv(0,1)$ is the velocity vector of a static target.

After pulse compression and phase compensation for the term due to the moving target (assuming $\alpha_{m}, Y_{m}$ and $X_{m}$ known), in the fast-time spatial domain the received echo is (see [7] for details)

$$
\begin{array}{r}
S_{c}\left(k_{u}, X\right)=R_{p}\left(X-X_{m}\right) A\left(k_{u}, \boldsymbol{\theta}\right) f_{m}+A\left(k_{u}, \boldsymbol{\theta}_{0}\right) \times \\
\sum_{n} f_{n} R_{p}\left(X-X_{n}\right) e^{-j \frac{k_{u}^{2}}{4 k}\left(X_{n}+\frac{X_{m}}{\alpha_{m}^{2}}\right)} e^{-j k_{u}\left(Y_{n}-\frac{Y_{m}}{\alpha_{m}}\right)},
\end{array}
$$

where $R_{p}(X)$ is the auto-correlation of the emitted pulse. The Fresnel approximation has been taken in deriving (9).

Expression (9) is the sum of the desired signal, due to a moving target, and the returns from the static ground, whose statistics are to be derived.

\subsection{Static ground signal statistics}

Define $\mathbf{S}=\left[S_{-N} \cdots S_{0} \cdots S_{N}\right]^{T}$, where $S_{i} \equiv S\left(k_{u_{i}}, X_{m}\right)$, $k_{u_{i}}=\frac{i}{2 N} \Delta K$, for $i=-N, \ldots, 0, \ldots, N$ and $\Delta K$ is the synthetic aperture bandwidth. Define also $\mathbf{A}(\boldsymbol{\theta})=\left[A_{-N}(\boldsymbol{\theta}) \cdots\right.$ $\left.A_{0}(\boldsymbol{\theta}) \cdots A_{N}(\boldsymbol{\theta})\right]$ where $A_{i}(\boldsymbol{\theta})=A\left(k_{u_{i}}, \boldsymbol{\theta}\right)$.

Let us assume for a while that the parameter $\boldsymbol{\theta}$ is known. In this case only the noise term in the expression (9) is random. Assuming that the number of static scatterers is large, none is predominant, and that they are uniformly distributed within a wavelength, then the complex noise term is circular symmetric and Gaussian [8]. Therefore, vector $\mathbf{S}$ density conditioned to $\boldsymbol{\theta}$ and $f_{m}$ is $p\left(\mathbf{S} \mid f_{m}, \boldsymbol{\theta}\right)=N\left(\mathbf{m}_{s}, \mathbf{C}_{s}\right)$ where $\mathbf{m}_{S}=E[\mathbf{S}]$ and $\mathbf{C}_{s}=E\left[\left(\mathbf{S}-\mathbf{m}_{s}\right)\left(\mathbf{S}-\mathbf{m}_{s}\right)^{H}\right]$.

In work [9] we show that $E[\mathbf{S}]=f_{m} \mathbf{A}(\boldsymbol{\theta})$ and $\mathbf{C}_{s}=$ $\eta L E_{p} \operatorname{diag}\left(\left|\left[A\left(\boldsymbol{\theta}_{0}\right)\right]_{i}\right|^{2}\right)$, for $i=-N, \ldots, N$, where $\eta$ is the mean power per unit of aera, $\mathrm{L}$ is the cross-range length, and $E_{p}$ is the energy of the transmitted pulse in range.

\subsection{Bayesian Approach}

We assume that both the components of the target velocity and the target reflectivity are independent, i.e.,

$$
p\left(\boldsymbol{\theta}, f_{m}\right)=p\left(\mu_{m}\right) p\left(\nu_{m}\right) p\left(f_{m}\right) .
$$

Furthermore, $\mu_{m}$ and $\nu_{m}$ are assumed to be uniformly distributed, whereas $f_{m}$ is assumed to be zero-mean, Gaussian complex circular distributed, with variance $\sigma_{a}$. Writing $f_{m}=\rho_{m} e^{j \phi_{m}}$, this assumption is equivalent to have uniform distribution on $\phi_{m}$ and Rayleigh distribution on $\rho_{m}$.

We do not have any prior information about the reflectivity of moving targets. The prior $p\left(f_{m}\right)$ should be therefore noninformative. This is the case with $\phi_{m}$, which is uniformly distributed. In order to have a noninformative prior on $\rho_{m}$ we take a very large $\sigma_{a}$.
The joint distribution of $\mathbf{S}, f_{m}$ and $\boldsymbol{\theta}$ is then given by

$$
p\left(\mathbf{S}, f_{m}, \boldsymbol{\theta}\right) \propto \frac{e^{-\left[\mathbf{S}-f_{m} \mathbf{A}(\theta)\right]^{H} \mathbf{C}_{s}^{-1}\left[\mathbf{S}-f_{m} \mathbf{A}(\theta)\right]-\frac{\left|f_{m}\right|^{2}}{\sigma_{a}^{2}}}}{2 \pi^{N}\left|\mathbf{C}_{s}\right| 2 \pi \sigma_{a}^{2}} .
$$

Equation (11) can be written as

$$
p\left(\mathbf{S}, f_{m}, \boldsymbol{\theta}\right) \propto \frac{\sigma^{2} e^{-\mathbf{C}}}{2 \pi^{N}\left|\mathbf{C}_{\mathbf{s}}\right| \sigma_{a}^{2}} \frac{e^{-\left(f_{m}-\bar{f}_{m}\right)^{H} \sigma^{-2}\left(f_{m}-\bar{f}_{m}\right)}}{2 \pi \sigma^{2}}
$$

where

$$
\begin{aligned}
\sigma^{-2} & =\sigma_{a}^{-2}+\mathbf{A}(\boldsymbol{\theta})^{H} \mathbf{C}_{s}^{-1} \mathbf{A}(\boldsymbol{\theta}) \\
\bar{f}_{m} & =\mathbf{A}(\boldsymbol{\theta})^{H} \mathbf{C}_{s}^{-1} \mathbf{S} \sigma^{2} ; \\
\mathbf{C} & =\mathbf{S}^{H} \mathbf{C}_{s}^{-1} \mathbf{S}-\left|\bar{f}_{m}\right|^{2} \sigma^{-2}
\end{aligned}
$$

Since $f_{m}$ is a nuisance parameter, we integrated it out. This poses no problem, since density $p\left(\mathbf{S}, f_{m}, \boldsymbol{\theta}\right)$ is complex Gaussian on $f_{m}$.

As estimation criterion we have adopted the the maximum a posteriori (MAP). By considering $\sigma_{a}$ very large, the MAP estimator for $\boldsymbol{\theta}$ is given by

$$
\begin{aligned}
\hat{\boldsymbol{\theta}}_{M A P}= & \arg \max _{\boldsymbol{\theta}}\left\{-\ln \left\{\mathbf{A}(\boldsymbol{\theta})^{H} \mathbf{C}_{s}^{-1} \mathbf{A}(\boldsymbol{\theta})\right\}\right. \\
& \left.+\frac{\left|\mathbf{A}(\boldsymbol{\theta})^{H} \mathbf{C}_{s}^{-1} \mathbf{S}\right|^{2}}{\mathbf{A}(\boldsymbol{\theta})^{H} \mathbf{C}_{s}^{-1} \mathbf{A}(\boldsymbol{\theta})}\right\} \\
= & \arg \max _{\boldsymbol{\theta}}\left\{-\ln \left(\sum_{i=-N}^{N} \frac{\left|A_{i}(\boldsymbol{\theta})\right|^{2}}{C_{s}(i, i)}\right)\right. \\
& \left.+\frac{\sum_{i=-N}^{N}\left|A_{i}(\boldsymbol{\theta})^{*} S_{i} C_{s}^{-1}(i, i)\right|^{2}}{\sum_{i=-N}^{N}\left|A_{i}(\boldsymbol{\theta})\right|^{2} C_{s}^{-1}(i, i)}\right\} .
\end{aligned}
$$

The overall scheme for detection, imaging and trajectory parameter estimation is summarized as follows:

- Detect and isolate regions (digital spotlight) which may contain moving targets;

- Estimate the corresponding $\alpha_{m}$ for each moving target;

- Image each target with its corresponding $\alpha_{m}$;

- Synthesize each target signature back to the original $\left(k_{u}, w\right)$ domain;

- Estimate the velocity components $\left(\mu_{m}, \nu_{m}\right)$ using equation (14).

The first four steps are described in [3]. 
Table 1. Mission parameters used in the simulation.

\begin{tabular}{|c|c|}
\hline Parameter & Value \\
\hline Carrier frequency & $5 \mathrm{GHz}$ \\
Chirp bandwidth & $150 \mathrm{MHz}$ \\
Altitude & $10 \mathrm{Km}$ \\
Velocity & $300 \mathrm{Km} / \mathrm{h}$ \\
Look angle & $20^{\circ}$ \\
Antenna radiation pattern & rectangular \\
Oversampling factor & 2 \\
\hline
\end{tabular}

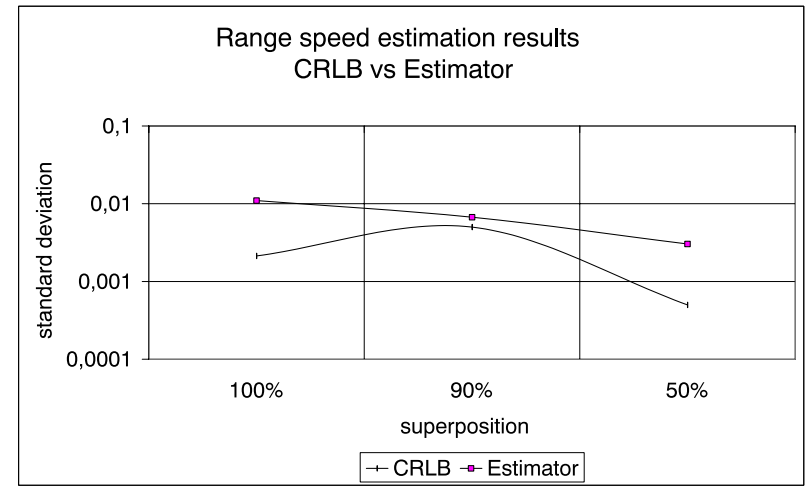

Fig. 4. Slant-range velocity CRLB (square root) and estimation results versus ground returns superposition).

\section{SIMULATION RESULTS}

In this section we apply the derived MAP estimator to simulated data. Table 1 displays the simulation parameters. A rectangular antenna shape has been used. In [10] we show that this shape leads to much better results than, for example, a Gaussian one.

Figures 4 and 5 plot the square root of the sample mean square error and the Cramèr-Rao lower bound (CRLB) relative to density $p\left(\boldsymbol{S} \mid f_{m}, \boldsymbol{\theta}\right)$, both as function of the percentage of superposition between the spectrum support of the static targets and of the moving target. The superposition is defined as $\left|K_{a}-k \mu_{m}\right| / K_{a}$, where $K_{a}$ is the $-3 \mathrm{~dB}$ antenna bandwidth. The signal to noise ratio $(S N R)$ was set to $\left|f_{m}\right|^{2} / \eta=1(S N R=0 \mathrm{~dB})$.

Comparing Fig.'s 4 and 5, we readily conclude that standard deviation of the slant-range velocity estimate is much lower than the standard deviation of the cross-range velocity estimate ; for the selected parameters, the former takes values close to 0.01 , whereas the latter takes values close to 1 . This is a consequence of the much higher sensitivity of $A\left(k_{u}, \mu, \nu\right)$ to $\mu$ than to $\nu$. However, even the low $\mathrm{SNR}=0 \mathrm{~dB}$, the quality of the azimuth velocity estimates is acceptable for many purposes.

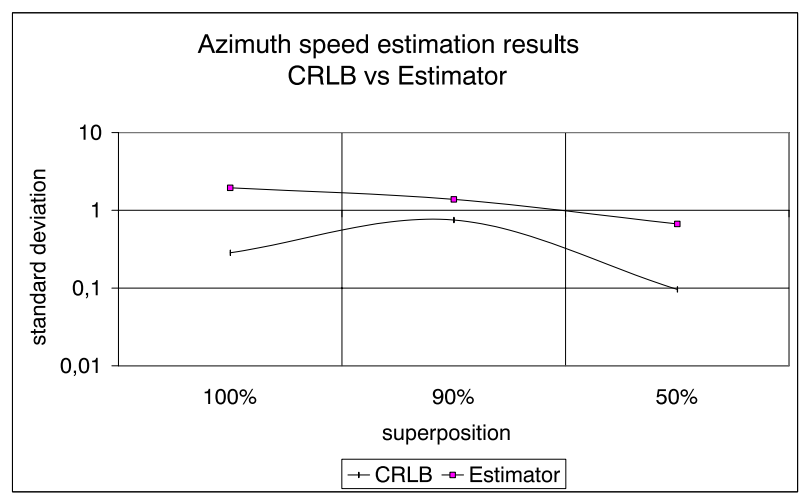

Fig. 5. Cross-range velocity CRLB (square root) and estimation results versus ground returns superposition).

\section{CONCLUDING REMARKS}

We presented a novel method to determine the complete velocity vector of a moving target using a single SAR sensor. Basically, we took advantage of the structure of the received signal: it is approximately a shifted and compressed version of the antenna radiation pattern. Each of these effects is mainly due to an orthogonal component of the velocity vector.

The algorithm is composed of two main parts: in the first part, the magnitude of the velocity targets is determined using the methodology proposed in [?]; in the second part, the blind angle ambiguity is solved by adopting a Bayesian approach to derive an estimator for the complete velocity vector.

As estimation criterion, we adopted the maximum a posteriori probability. The estimator performance was illustrated by using simulated data. We concluded that for a typical airborne scenario using a C-band radar and a $S N R=$ $0 \mathrm{~dB}$, the the standard deviation of the range velocity takes values close to 0.01 , whereas the standard deviation of the azimuth velocity takes values close to 1 .

\section{REFERENCES}

[1] Kazuo Ouchi, "On the multilook images of moving targets by synthetic aperture radars", IEEE Transactions on Antennas and Propagation, vol. AP-33, pp. 823-827, August 1985.

[2] Martin Kirsht, "Detection, velocity estimation and imaging of moving targets with single-channel sar", in Proc. of the EUSAR'98, pp. 587-590, 1998.

[3] Mehrdad Soumekh, "Reconnaissance with ultra wideband uhf synthetic aperture radar", IEEE Signal Processing Magazine, vol. , pp. 21-40, July 1995.

[4] Mehrdad Soumekh, Synthetic Aperture Radar Signal Processing with MATLAB algorithms, WILEYINTERSCIENCE, 1999. 
[5] Mehrdad Soumekh, Fourier Array Imaging, Prentice Hall, 1994.

[6] Robert E. Collin, Antennas and Radiowave Propagation, McGraw Hill, 1987.

[7] Paulo Marques and José Dias, "Optimal detection and imaging of moving objects with unknown velocity", in Proc. of the 3rd European Conference on Synthetic Aperture Radar, 2000.

[8] C. Jakowatz, D. Wahl, P. Eichel, D. Ghiglia, and P. Thompson, Spotlight-Mode Synthetic Aperture Radar: A Signal Processing Approach, Kluwer Academic Publishers, Boston, 1996.

[9] Paulo Marques and José Dias, "Blind angle ambiguity resolution in parameter estimation of moving targets using a single sar sensor", in Proc. of the EUSIPCO'2000 (to be published), 2000.

[10] Paulo Marques and José Dias, "Optimal detection and imaging of moving objects with unknown velocity", in Proc. of the International Geoscience and Remote Sensing Symposium, 2000. 